\title{
Is there a novel Einstein-Gauss-Bonnet theory in four dimensions?
}

\author{
Metin Gürses ${ }^{1, a}$, Tahsin Çağrı Şişman ${ }^{2, b}$, Bayram Tekin ${ }^{3, c}$ \\ ${ }^{1}$ Department of Mathematics, Faculty of Sciences, Bilkent University, 06800 Ankara, Turkey \\ 2 Department of Astronautical Engineering, University of Turkish Aeronautical Association, 06790 Ankara, Turkey \\ ${ }^{3}$ Department of Physics, Middle East Technical University, 06800 Ankara, Turkey
}

Received: 12 May 2020 / Accepted: 2 July 2020 / Published online: 20 July 2020

(C) The Author(s) 2020

\begin{abstract}
No! We show that the field equations of EinsteinGauss-Bonnet theory defined in generic $D>4$ dimensions split into two parts one of which always remains higher dimensional, and hence the theory does not have a non-trivial limit to $D=4$. Therefore, the recently introduced fourdimensional, novel, Einstein-Gauss-Bonnet theory does not admit an intrinsically four-dimensional definition, in terms of metric only, as such it does not exist in four dimensions. The solutions (the spacetime, the metric) always remain $D>4$ dimensional. As there is no canonical choice of 4 spacetime dimensions out of $D$ dimensions for generic metrics, the theory is not well defined in four dimensions.
\end{abstract}

\section{Introduction}

Recently a four-dimensional Einstein-Gauss-Bonnet theory that is claimed to propagate only a massless spin- 2 graviton was introduced as the $D \rightarrow 4$ limit in [1] with the action

$$
\begin{aligned}
I= & \int d^{D} x \sqrt{-g}\left[\frac{1}{\kappa}\left(R-2 \Lambda_{0}\right)\right. \\
& \left.+\frac{\alpha}{D-4}\left(R_{\alpha \beta \rho \sigma} R^{\alpha \beta \rho \sigma}-4 R_{\alpha \beta} R^{\alpha \beta}+R^{2}\right)\right],
\end{aligned}
$$

of which the field equations are [2,3]

$$
\frac{1}{\kappa}\left(R_{\mu \nu}-\frac{1}{2} g_{\mu \nu} R+\Lambda_{0} g_{\mu \nu}\right)+\frac{\alpha}{D-4} \mathcal{H}_{\mu \nu}=0
$$

\footnotetext{
a e-mail: gurses@fen.bilkent.edu.tr

b e-mail: tahsin.c.sisman@gmail.com

c e-mail: btekin@metu.edu.tr (corresponding author)
}

where the "Gauss-Bonnet tensor" reads

$$
\begin{aligned}
\mathcal{H}_{\mu \nu}= & 2\left[R R_{\mu \nu}-2 R_{\mu \alpha \nu \beta} R^{\alpha \beta}+R_{\mu \alpha \beta \sigma} R_{v}{ }^{\alpha \beta \sigma}-2 R_{\mu \alpha} R_{v}^{\alpha}\right. \\
& \left.-\frac{1}{4} g_{\mu \nu}\left(R_{\alpha \beta \rho \sigma} R^{\alpha \beta \rho \sigma}-4 R_{\alpha \beta} R^{\alpha \beta}+R^{2}\right)\right]
\end{aligned}
$$

For $D>4,(2)$ is the well-known Einstein-Gauss-Bonnet theory which has been studied in the literature in great detail. On the other hand, for $D=4$, the $\mathcal{H}_{\mu \nu}$ tensor vanishes identically and hence, as per common knowledge, the field equations (2) reduces to the Einstein's theory. This is because in four dimensions, the Gauss-Bonnet combination $\mathcal{G}:=R_{\alpha \beta \rho \sigma} R^{\alpha \beta \rho \sigma}-4 R_{\alpha \beta} R^{\alpha \beta}+R^{2}$ can be written as $\mathcal{G}=\epsilon_{\mu \nu \alpha \beta} \epsilon^{\mu \nu \sigma \rho} R^{\alpha \beta \gamma \lambda} R_{\gamma \lambda \sigma \rho}$ and yields a topological action, i.e. the Euler number which is independent of the metric $g_{\mu \nu}$. This was the state of affairs until the paper [1] implicitly asked the question "how does the $\mathcal{H}_{\mu \nu}$ tensor go to zero as $D \rightarrow 4$ ?". The answer is very interesting: because if it goes to zero in the following way

$\mathcal{H}_{\mu \nu}=(D-4) \mathcal{Y}_{\mu \nu}$

where $\mathcal{Y}_{\mu \nu}$ is a new tensor to be found, then the authors of [1] argue that in the $D \rightarrow 4$ limit, the field equations (2) define a four-dimensional theory in the limit. So namely, the suggested four-dimensional theory would be the following theory in source-free case:

$\lim _{D \rightarrow 4}\left[\frac{1}{\kappa}\left(R_{\mu \nu}-\frac{1}{2} g_{\mu \nu} R+\Lambda_{0} g_{\mu \nu}\right)+\frac{\alpha}{D-4} \mathcal{H}_{\mu \nu}\right]=0$.

Let us try to understand what the suggested theory is. As there is no intrinsically defined four-dimensional covariant tensor that the Gauss-Bonnet tensor reduces to; namely, $\mathcal{Y}_{\mu \nu}$ 
in (4) does not exist as guaranteed by the Lovelock theorem [4-6] and as will be shown below, the theory must be defined as a limit. Thus, to compute anything in this theory, say the perturbative particle content, the maximally symmetric vacua, the black hole solutions, or any solution with or without a symmetry, one must do the computation in $D$ dimensions and than take the $D \rightarrow 4$ limit. Surely, for some components of the the metric such as the spherically symmetric metric, due to the nature of the the Gauss-Bonnet tensor, this limit might make sense. But, at the level of the solutions, namely at the level of the full metric, this limit makes no sense at all. For example, assume that there is a solution to the theory given locally with the $D$ dimensional metric $g_{\mu \nu}$ say which has no isometries. Then, as we need to take the $D \rightarrow 4$ limit, which dimensions or coordinates do we dispose of, is there a canonical prescription? The answer is no! Even for spherically symmetric solutions of Boulware and Deser [7], studied so far, we do not have the right to dispose any dimension we choose.

What we have just stated is actually at the foundations of defining a gravity theory in the Riemannian geometry context. The Riemannian geometry depends on the number of dimensions, in defining a classical gravity theory based on geometry one first fixes the number of dimensions to be some $D$; and as this number changes, the theory changes. There is no sensible limiting procedure as defined by (5); there is of course compactification, dimensional reduction etc where all the spacetime dimensions still survive albeit not in equal sizes generically.

The layout of the paper is as follows: in Sect. 2, we recast the $D$-dimensional Gauss-Bonnet tensor using the Weyl tensor in such a way that it naturally splits into two parts. One part has a formal $D \rightarrow 4$ limit, while the other part is always higher dimensional. In Sect. 3, we give another proof that the theory is non-trivial only for $D>4$ using the first-order formalism with the vielbein and the spin-connection. In Sect. 4, we give a an explicit example in the form of a direct-product metric where the role of the higher dimensional part is apparent.

\section{$2 D \rightarrow 4$ limit of the field equations}

To further lay out our ideas, and to show that there is no fourdimensional definition of the theory, let us recast the GaussBonnet tensor, in such a way that we can see the limiting behaviors. For this purpose, the Weyl tensor,

$$
\begin{aligned}
C_{\mu \alpha \nu \beta}= & R_{\mu \alpha \nu \beta}-\frac{2}{(D-2)}\left(g_{\mu[\nu} R_{\beta] \alpha}-g_{\alpha[\nu} R_{\beta] \mu}\right) \\
& +\frac{2}{(D-1)(D-2)} R g_{\mu[\nu} g_{\beta] \alpha},
\end{aligned}
$$

becomes rather useful. Using Appendix A of [8], the GaussBonnet tensor in $D$ dimensions can be split as

$\mathcal{H}_{\mu \nu}=2\left(\mathcal{L}_{\mu \nu}+\mathcal{Z}_{\mu \nu}\right)$

where the first term does not have an explicit coefficient related to the number of dimensions and is given as

$\mathcal{L}_{\mu \nu}:=C_{\mu \alpha \beta \sigma} C_{\nu}{ }^{\alpha \beta \sigma}-\frac{1}{4} g_{\mu \nu} C_{\alpha \beta \rho \sigma} C^{\alpha \beta \rho \sigma}$,

which we shall name as the Lanczos-Bach tensor, and the other part carries explicit coefficients regarding the number of dimensions:

$$
\begin{aligned}
\mathcal{Z}_{\mu \nu}:= & \frac{(D-4)(D-3)}{(D-1)(D-2)}\left[-\frac{2(D-1)}{(D-3)} C_{\mu \rho \nu \sigma} R^{\rho \sigma}\right. \\
& -\frac{2(D-1)}{(D-2)} R_{\mu \rho} R_{\nu}^{\rho}+\frac{D}{(D-2)} R_{\mu \nu} R \\
& \left.+\frac{1}{(D-2)} g_{\mu \nu}\left((D-1) R_{\rho \sigma} R^{\rho \sigma}-\frac{D+2}{4} R^{2}\right)\right]
\end{aligned}
$$

where we kept all the factors to see how the limiting procedure might work. With the $2 /(D-4)$ factor, the second part nicely reduces to a tensor $\mathcal{S}_{\mu \nu}$ which is finite in the $D \rightarrow 4$ limit;

$\mathcal{S}_{\mu \nu}:=\frac{2}{D-4} \mathcal{Z}_{\mu \nu}$

But, the first part is rather non-trivial. In $D=4$ dimensions we have the Lanczos-Bach identity [6,9] for any smooth metric;

$$
C_{\mu \alpha \beta \sigma} C_{\nu}{ }^{\alpha \beta \sigma}=\frac{1}{4} g_{\mu \nu} C_{\alpha \beta \rho \sigma} C^{\alpha \beta \rho \sigma}
$$

for all metrics in $D=4$.

Thus, a cursory look might suggest that one might naively assume the Lanczos-Bach identity in four dimensions and set $\mathcal{L}_{\mu \nu}=0$ in the $D \rightarrow 4$ limit yielding a finite intrinsically four dimensional description of the Gauss-Bonnet tensor as

$$
\begin{aligned}
& \lim _{D \rightarrow 4}\left(\frac{1}{D-4} \mathcal{H}_{\mu \nu}\right)=\frac{1}{3}\left[-6 C_{\mu \rho \nu \sigma} R^{\rho \sigma}-3 R_{\mu \rho} R_{\nu}^{\rho}\right. \\
& \left.\quad+2 R_{\mu \nu} R+\frac{3}{2} g_{\mu \nu}\left(R_{\rho \sigma} R^{\rho \sigma}-\frac{1}{2} R^{2}\right)\right] .
\end{aligned}
$$

where the right-hand side is $\mathcal{S}_{\mu \nu}$, given in (10), calculated at $D=4$. But this is a red-herring! The $\mathcal{H}$ tensor or the $\mathcal{S}$ tensor does not obey the Bianchi identity

$\nabla^{\mu} \mathcal{S}_{\mu \nu} \neq 0$ 
Therefore, without further assumptions, it cannot be used in the description of a four dimensional theory. Then, this begs the question: How does the $\mathcal{L}_{\mu \nu}$ tensor go to zero in the $D \rightarrow 4$ limit, that is

$\lim _{D \rightarrow 4}\left[\frac{1}{D-4}\left(C_{\mu \alpha \beta \sigma} C_{\nu}{ }^{\alpha \beta \sigma}-\frac{1}{4} g_{\mu \nu} C_{\alpha \beta \rho \sigma} C^{\alpha \beta \rho \sigma}\right)\right]=?$

To save the Bianchi identity, $\mathcal{L}_{\mu \nu}$ should have the form

$\frac{2}{D-4} \mathcal{L}_{\mu \nu}=\mathcal{T}_{\mu \nu}$ for $D \neq 4$

If this is the case, then there is a discontinuity for the GaussBonnet tensor as

$\frac{1}{D-4} \mathcal{H}_{\mu \nu}= \begin{cases}\mathcal{T}_{\mu \nu}+\mathcal{S}_{\mu \nu}, & \text { for } D \neq 4, \\ \frac{0}{0}, & \text { for } D=4\end{cases}$

Then, in the $D \rightarrow 4$ limit, the Gauss-Bonnet tensor with an $\alpha / D-4$ factor becomes

$\lim _{D \rightarrow 4}\left(\frac{1}{D-4} \mathcal{H}_{\mu \nu}\right)=\mathcal{T}_{\mu \nu}+\mathcal{S}_{\mu \nu}$,

that is the Gauss-Bonnet tensor is not continuous in $D$ at $D=$ 4. This discontinuity in the Gauss-Bonnet tensor introduces a problem: Let $g_{\mu \nu}^{D}$ be the solution of the field equations for $D>4$, and $g_{\mu \nu} \lim$ is the solution of the limiting field equations (17); then

$\lim _{D \rightarrow 4} g_{\mu \nu}^{D} \neq g_{\mu \nu}$,

in general.

Incidentally, the $\mathcal{L}_{\mu \nu}$ tensor is related to the trace of the $D$ dimensional extension of the Bel-Robinson tensor given in [10] which reads

$$
\begin{aligned}
\mathcal{B}_{\alpha \beta \lambda \mu}= & C_{\alpha \rho \lambda \sigma} C_{\beta}{ }^{\rho}{ }_{\mu}{ }^{\sigma}+C_{\alpha \rho \mu \sigma} C_{\beta}{ }^{\rho}{ }_{\lambda}{ }^{\sigma} \\
& -\frac{1}{2} g_{\alpha \beta} C_{\rho \nu \lambda \sigma} C^{\rho \nu}{ }_{\mu}{ }^{\sigma} \\
& -\frac{1}{2} g_{\lambda \mu} C_{\alpha \rho \sigma \nu} C_{\beta}{ }^{\rho \sigma \nu}+\frac{1}{8} g_{\alpha \beta} g_{\lambda \mu} C_{\rho \nu \sigma \eta} C^{\rho \nu \sigma \eta},
\end{aligned}
$$

and one has

$$
g^{\lambda \mu} \mathcal{B}_{\alpha \beta \lambda \mu}=\frac{D-4}{2} \mathcal{L}_{\alpha \beta}
$$

To summarize, in this section, we have shown that there is a part of the Gauss-Bonnet tensor $\mathcal{H}_{\mu \nu}$ which is always higher dimensional even though one part of the tensor can be made finite with the procedure of dividing by $1 /(D-4)$ and then formally assuming that the remaining indices are four dimensional. The all important point here is that if one bluntly drops the extra dimensional part (which we called $\mathcal{L}_{\mu \nu}$ ), then the Bianchi identity is not satisfied for the remaining fourdimensional theory. Thus, one either has all the dimensions, or one has four dimensions without the benefit of the Bianchi identity. If one chooses the second option, one cannot couple the four-dimensonal theory to conserved matter fields; or one must impose the Bianchi identity on-shell for the solutions.

\section{The field equations in first-order formulation}

The authors of [1] argued that in the first-order formulation of the Gauss-Bonnet theory a $(D-4)$ factor arises in the field equations, and this factor can be canceled by introducing the $\alpha /(D-4)$ factor in the action. This claim needs to be scrutinized carefully as we do here. Let us just consider the Gauss-Bonnet part of the action without any factors or coefficients. Then, we have the $D$-dimensional action in terms of the vielbein 1-form $e^{a}$ and the curvature 2-form $R^{a b}:=d \omega^{a b}+\omega^{a c} \wedge \omega_{c}^{b}$

$I_{G B}=\int_{\mathcal{M}_{D}} \epsilon_{a_{1} a_{2} \cdots a_{D}} R^{a_{1} a_{2}} \wedge R^{a_{3} a_{4}} \wedge e^{a_{5}} \wedge e^{a_{6}} \cdots \wedge e^{a_{D}}$

where the Latin indices refer to the tangent frame. Then, varying the action with respect to the spin connection yields zero in the zero torsion case; and the rest of the field equations are obtained by varying with respect to the vielbein. At this stage the discussion bifurcates: ${ }^{1}$ Assume that $D=4$, then the action reduces to $\int_{\mathcal{M}_{4}} \epsilon_{a_{1} a_{2} a_{3} a_{4}} R^{a_{1} a_{2}} \wedge R^{a_{3} a_{4}}$ where there is no vierbein left and one has

$\delta_{e^{a}} \int_{\mathcal{M}_{4}} \epsilon_{a_{1} a_{2} a_{3} a_{4}} R^{a_{1} a_{2}} \wedge R^{a_{3} a_{4}}=0, \quad D=4$.

On the other hand, for generic $D>4$ dimensions, variation with respect to the vielbein yields the field equation as a $(D-1)$-form

$$
\begin{aligned}
& \mathcal{E} a_{D}=(D-4) \epsilon_{a_{1} a_{2} \cdots a_{D}} R^{a_{1} a_{2}} \wedge R^{a_{3} a_{4}} \wedge e^{a_{5}} \wedge e^{a_{6}} \\
& \quad \cdots \wedge e^{a_{D-1}} \quad D>4 .
\end{aligned}
$$

Clearly the $(D-4)$ factor arises, but it does so only in $D$ dimensions: one cannot simply multiply with a $\alpha /(D-4)$ and take the $D \rightarrow 4$ limit! In fact, starting from the last equation, one can get the second order, metric form of the Gauss-Bonnet tensor $\mathcal{H}_{\mu \nu}$, and in the process, one sees the role played by this $(D-4)$ factor. To do so, instead of the

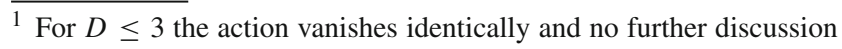
is needed. 
tangent frame indices we can recast it in terms of the spacetime indices as which can be written as

$$
\begin{aligned}
\mathcal{E}_{\nu}= & \frac{(D-4)}{4} \epsilon_{\mu_{1} \mu_{2} \ldots \mu_{D-1} \nu} R^{\mu_{1} \mu_{2}}{ }_{\sigma_{1} \sigma_{2}} R^{\mu_{3} \mu_{4}}{ }_{\sigma_{3} \sigma_{4}} d x^{\sigma_{1}} \ldots \\
& \times \wedge d x^{\sigma_{4}} \wedge d x^{\mu_{5}} \ldots \wedge d x^{\mu_{D-1}} .
\end{aligned}
$$

This is really a covariant vector-valued $(D-1)$-form, and the Hodge dual of this $(D-1)$-form is a 1-form; and since we have

$$
\begin{aligned}
* & \left(d x^{\sigma_{1}} \ldots \wedge d x^{\sigma_{4}} \wedge d x^{\mu_{5}} \ldots \wedge d x^{\mu_{D-1}}\right) \\
= & \epsilon^{\sigma_{1} \ldots \sigma_{4} \mu_{5} \ldots \mu_{D-1}} \mu_{D} d x^{\mu_{D}},
\end{aligned}
$$

the 1-form field equations read

$$
\begin{gathered}
* \mathcal{E}_{\nu}=\frac{(D-4)}{4} \epsilon_{\mu_{1} \mu_{2} \ldots \mu_{D-1} \nu} \epsilon^{\sigma_{1} \ldots \sigma_{4} \mu_{5} \ldots \mu_{D-1}} \mu_{D} \\
R_{\sigma_{1} \sigma_{2}}^{\mu_{1} \mu_{2}} R_{\sigma_{3} \sigma_{4}}^{\mu_{3} \mu_{4}} d x^{\mu_{D}},
\end{gathered}
$$

from which we define the rank-2 tensor $\mathcal{E}_{v \alpha}$ as

$* \mathcal{E}_{v}=: \mathcal{E}_{v \alpha} d x^{\alpha}$.

Explicitly one has

$\mathcal{E}_{\nu \alpha}=\frac{(D-4)}{4} \epsilon_{\mu_{1} \mu_{2} \ldots \mu_{D-1} \nu} \epsilon^{\sigma_{1} \ldots \sigma_{4} \mu_{5} \ldots \mu_{D-1}}{ }_{\alpha} R^{\mu_{1} \mu_{2}}{ }_{\sigma_{1} \sigma_{2}} R^{\mu_{3} \mu_{4}}{ }_{\sigma_{3} \sigma_{4}}$,

which can be further reduced with the help of the identity

$\epsilon_{\mu_{1} \mu_{2} \ldots \mu_{D-1} \nu} \epsilon^{\sigma_{1} \ldots \sigma_{4} \mu_{5} \ldots \mu_{D-1}} \alpha=-(D-5) ! g_{\beta \alpha} \delta_{\mu_{1} \ldots \mu_{4} v}^{\sigma_{1} . \sigma_{4} \beta}$,

where we used the generalized Kronecker delta. So, we have

$$
\begin{aligned}
\mathcal{E}_{v \alpha} & =-\frac{(D-4)}{4}(D-5) ! g_{\beta \alpha} \delta_{\mu_{1} \ldots \mu_{4} \nu}^{\sigma_{1} . \sigma_{4} \beta} R_{\sigma_{1} \sigma_{2}}^{\mu_{1} \mu_{2}} R_{\sigma_{3} \sigma_{4}}^{\mu_{3} \mu_{4}} \\
& =-\frac{(D-4) !}{4} g_{\beta \alpha} \delta_{\mu_{1} \ldots \mu_{4} \nu}^{\sigma_{1} . \sigma_{4} \beta} R_{\sigma_{1} \sigma_{2}}^{\mu_{1} \mu_{2}} R_{\sigma_{3} \sigma_{4}}^{\mu_{3} \mu_{4}}
\end{aligned}
$$

Observe that the $(D-4)$ factor turned into $(D-4)$ ! which does not vanish for $D=4$. Since one also has

$$
g_{\beta \alpha} \delta_{\mu_{1} \ldots \mu_{4} \nu}^{\sigma_{1} . . \sigma_{4} \beta} R_{\sigma_{1} \sigma_{2}}^{\mu_{1} \mu_{2}} R_{\sigma_{3} \sigma_{4}}^{\mu_{3} \mu_{4}}=-8 \mathcal{H}_{\nu \alpha}
$$

where $\mathcal{H}_{v \alpha}$ is the Gauss-Bonnet tensor we defined above, we get

$\mathcal{E}_{v \mu}=2(D-4) ! \mathcal{H}_{v \alpha}$

Thus, the moral of the story is that one either has an explicit $(D-4)$ factor in front of the field equations when they are written in terms of the vielbeins and the spin connection where the dimensionality of the spacetime is explicitly $D>$ 4 as counted by the number of vielbeins; or, one does not have an explicit $(D-4)$ factor in the field equations in the metric formulation. There is no other option. In the metric formulation, we have shown in the previous section that a $(D-4)$ does not arise for generic metrics in all parts of the field equations.

\section{Direct-product spacetimes}

To see the alluded problems explicitly in an example, let us consider the direct-product spacetimes for which the $D$ dimensional metric has the form

$$
\begin{aligned}
d s^{2}= & g_{A B} d x^{A} d x^{B}=g_{\alpha \beta}\left(x^{\mu}\right) d x^{\alpha} d x^{\beta} \\
& +g_{a b}\left(x^{c}\right) d x^{a} d x^{b},
\end{aligned}
$$

where $A, B=1,2 \ldots, D ; \alpha, \beta=1,2,3,4$; and $a, b=$ $5,6, \cdots, D$. Here, $g_{\alpha \beta}$ depends only on the four-dimensional coordinates $x^{\mu}$, and $g_{a b}$ depends only on the extra dimensional coordinates $x^{c}$. Then, for the Christoffel connection,

$\Gamma_{B C}^{A}=\frac{1}{2} g^{A E}\left(\partial_{B} g_{E C}+\partial_{C} g_{E B}-\partial_{E} g_{B C}\right)$,

it is easy to show that the only nonzero parts are

$$
\begin{gathered}
{ }_{D} \Gamma_{\beta \mu}^{\alpha}={ }_{4} \Gamma_{\beta \mu}^{\alpha}=\frac{1}{2} g^{\alpha \epsilon}\left(\partial_{\beta} g_{\mu \epsilon}+\partial_{\mu} g_{\beta \epsilon}-\partial_{\epsilon} g_{\beta \mu}\right), \\
{ }_{D} \Gamma_{b c}^{a}={ }_{d} \Gamma_{b c}^{a}=\frac{1}{2} g^{a e}\left(\partial_{b} g_{c e}+\partial_{c} g_{b e}-\partial_{e} g_{b c}\right)
\end{gathered}
$$

where the subindex $d$ denotes the $(D-4)$-dimensional. Due to this property, we have the following nonzero components of the Riemann tensor, $R_{B C E}^{A}$, and Ricci tensor, $R_{A B}$;

$$
\begin{aligned}
{ }_{D} R_{\beta \mu \epsilon}^{\alpha} & ={ }_{4} R_{\beta \mu \epsilon}^{\alpha}, \quad{ }_{D} R_{b c e}^{a}={ }_{d} R_{b c e}^{a}, \\
D & R_{\alpha \beta}={ }_{4} R_{\alpha \beta}, \quad D R_{a b}={ }_{d} R_{a b} .
\end{aligned}
$$

In addition, the scalar curvature splits as

${ }_{D} R={ }_{4} R+{ }_{d} R$.

The nonzero components of the Weyl tensor $C_{A B E F}$ are

$$
\begin{aligned}
& { }_{D} C_{\alpha \beta \epsilon \nu}={ }_{4} C_{\alpha \beta \epsilon \nu}+\frac{(D-4)}{(D-2)}\left(g_{\alpha\left[\epsilon{ }_{4}\right.} R_{\nu] \beta}-g_{\beta[\epsilon 4} R_{\nu] \alpha}\right) \\
& -\frac{(D-4)(D+1)}{3(D-1)(D-2)} 4 \operatorname{Rg}_{\alpha[\epsilon} g_{\nu] \beta} \\
& +\frac{2}{(D-1)(D-2)} d{ }_{d} g_{\alpha[\epsilon} g_{\nu] \beta}, \\
& { }_{D} C_{\text {abef }}={ }_{d} C_{\text {abef }}+\frac{8}{(D-2)(D-6)} \\
& \times\left(g_{a[e} R_{f] b}-g_{b[e d} R_{f] a}\right)
\end{aligned}
$$




$$
\begin{aligned}
& -\frac{8(2 D-7)}{(D-1)(D-2)(D-5)(D-6)}{ }_{d} R g_{a[e} g_{f] b} \\
& +\frac{2}{(D-1)(D-2)}{ }_{4} R g_{a[e} g_{f] b}, \\
{ }_{D} C_{\alpha b a \beta}= & \frac{1}{(D-2)}\left(g_{\alpha \beta d} R_{a b}+g_{a b}{ }_{4} R_{\alpha \beta}\right) \\
& -\frac{1}{(D-1)(D-2)}\left({ }_{4} R+{ }_{d} R\right) g_{\alpha \beta} g_{a b},
\end{aligned}
$$

in addition to ${ }_{D} C_{\alpha b \beta a}=-{ }_{D} C_{b \alpha \beta a}={ }_{D} C_{b \alpha a \beta}=-{ }_{D} C_{\alpha b a \beta}$.

If the $d$-dimensional internal space is flat as

$d s^{2}=g_{A B} d x^{A} d x^{B}=g_{\alpha \beta}\left(x^{\mu}\right) d x^{\alpha} d x^{\beta}+\eta_{a b} d x^{a} d x^{b}$,

then one has

${ }_{D} R^{a}{ }_{b c e}=0, \quad{ }_{D} R_{a b}=0, \quad{ }_{D} R={ }_{4} R$,

and nonzero components of the Weyl tensor given in (39-41) become

$$
\begin{aligned}
{ }_{D} C_{\alpha \beta \epsilon \nu}= & { }_{4} C_{\alpha \beta \epsilon \nu}+\frac{(D-4)}{(D-2)}\left(g_{\alpha[\epsilon}{ }_{4} R_{\nu] \beta}-g_{\beta[\epsilon} R_{\nu] \alpha}\right) \\
& -\frac{(D-4)(D+1)}{3(D-1)(D-2)}{ }_{4} R g_{\alpha[\epsilon} g_{\nu] \beta}, \\
{ }_{D} C_{a b e f}= & \frac{2}{(D-1)(D-2)}{ }_{4} R \eta_{a[e} \eta_{f] b}, \\
{ }_{D} C_{\alpha b a \beta}= & \frac{1}{(D-2)} \eta_{a b}{ }_{4} R_{\alpha \beta}-\frac{1}{(D-1)(D-2)}{ }_{4} R g_{\alpha \beta \beta} \eta_{a b},
\end{aligned}
$$

in addition to ${ }_{D} C_{\alpha b \beta a}=-{ }_{D} C_{b \alpha \beta a}={ }_{D} C_{b \alpha a \beta}={ }_{D} C_{\alpha b a \beta}$.

With the above results, let us provide a clear example of where the limit

$$
\begin{aligned}
\lim _{D \rightarrow 4} \mathcal{L}_{A B}= & \lim _{D \rightarrow 4}\left[\frac { 1 } { D - 4 } \left(C_{A E F G} C_{B}^{E F G}\right.\right. \\
& \left.\left.-\frac{1}{4} g_{A B} C_{E F G H} C^{E F G H}\right)\right],
\end{aligned}
$$

fails. Consider the $\mathcal{L}_{a b}$ components of the Lanczos-Bach tensor,

$$
\mathcal{L}_{a b}=C_{a E F G} C_{b}^{E F G}-\frac{1}{4} \eta_{a b} C_{E F G H} C^{E F G H} .
$$

These components can be written as

$$
\begin{aligned}
\mathcal{L}_{a b}= & \left({ }_{D} C_{a e f g D} C_{b}{ }^{e f g}+2{ }_{D} C_{a \epsilon f \gamma D} C_{b}{ }^{\epsilon f \gamma}\right) \\
& -\frac{1}{4} \eta_{a b}\left({ }_{D} C_{\alpha \epsilon \nu \gamma D} C^{\alpha \epsilon \nu \gamma}+{ }_{D} C_{a e f g D} C^{a e f g}\right. \\
& \left.+4{ }_{D} C_{\alpha e \gamma f D} C^{\alpha e \gamma f}\right) .
\end{aligned}
$$

By using (44-46), the $\mathcal{L}_{a b}$ components of the Lanczos-Bach tensor can be calculated in terms of the four-dimensional and $d$-dimensional quantities as

$$
\begin{aligned}
\mathcal{L}_{a b}= & -\frac{1}{4} \eta_{a b}\left({ }_{4} C_{\epsilon \nu \gamma \eta} C_{4}{ }^{\epsilon \nu \gamma \eta}\right. \\
& +\frac{2\left(D^{2}-6 D+4\right)}{(D-2)^{2}}{ }_{4} R_{\epsilon \nu}{ }_{4} R^{\epsilon \nu} \\
& \left.-\frac{\left(D^{3}-5 D^{2}+2 D-16\right)}{3(D-1)(D-2)^{2}}{ }_{4} R^{2}\right) .
\end{aligned}
$$

Then, the $D \rightarrow 4$ limit for this term in the form,

$$
\begin{aligned}
& \lim _{D \rightarrow 4}\left[-\frac{\eta_{a b}}{4(D-4)}\left({ }_{4} C_{\epsilon \nu \gamma \eta} C_{4} C^{\epsilon \nu \eta}\right.\right. \\
& +\frac{2\left(D^{2}-6 D+4\right)}{(D-2)^{2}}{ }_{4} R_{\epsilon \nu}{ }_{4} R^{\epsilon \nu} \\
& \left.\left.-\frac{\left(D^{3}-5 D^{2}+2 D-16\right)}{3(D-1)(D-2)^{2}}{ }_{4} R^{2}\right)\right],
\end{aligned}
$$

is undefined, and this fact indicates that in general, there is no proper $D \rightarrow 4$ limit for the field equations for the directproduct spacetimes.

\section{Conclusions}

Recently [1], contrary to common knowledge and to the Lovelock's theorem [4-6], a novel four-dimensional EinsteinGauss-Bonnet theory with only a massless spin-2 graviton degree of freedom was suggested to exist. A fourdimensional gravity theory should have four-dimensional equations: here, we have shown that this is not the case. Namely, we have shown that the novel Einstein-GaussBonnet theory in four dimensions does not have an intrinsically four-dimensional description in terms of a covariantlyconserved rank-2 tensor in four dimensions. We have done this by splitting the Gauss-Bonnet tensor (2) into two parts as (7): one is what we called the Lanczos-Bach tensor (8) which is related to the trace of the $D$-dimensional Bel-Robinson tensor which does not have an explicit $(D-4)$ factor, and the other part (9) is a part that has an explicit $(D-4)$ factor in front. The Lanczos-Bach tensor vanishes identically in four dimensions; however, it cannot be set to identically zero in that dimensions since in the absence of it, the GaussBonnet tensor does not satisfy the Bianchi Identity. Thus, the theory must be defined in $D>4$ dimensions to be nontrivial which is in complete agreement with the Lovelock's theorem. But, once the theory is defined in $D$ dimensions, it will have all sorts of $D$ dimensional solutions and in none of these solutions one can simply dispose of $(D-4)$ dimensions or coordinates as such a discrimination among spacetime dimensions simply does not make sense. We gave an 
explicit example in the form of a direct product. In the firstorder formulation with the vielbein and the spin connection, there is an explicit $(D-4)$ factor in front of the field equations, but this factor only arises in $D>4$ dimensions not in four dimensions. What we have shown here for the GaussBonnet tensor in its critical $D=4$ dimensions is also valid for the other Lovelock tensors in their critical dimensions. As a final remark, let us note that following the $D \rightarrow 2$ formal limit of Einstein's gravity as was done in [11], if one carries out a $D \rightarrow 4$ construction in the EGB theory, one finds [1214], complementing our arguments, that the ensuing theory is not only a theory of massless spin-2 gravitons but additional scalar fields (of the Horndeski or Galileon type) appear.

Acknowledgements We would like to thank S. Deser and Y. Pang for useful discussions.

Data Availability Statement This manuscript has no associated data or the data will not be deposited. [Authors' comment: This work does not contain any data related analysis.]

Open Access This article is licensed under a Creative Commons Attribution 4.0 International License, which permits use, sharing, adaptation, distribution and reproduction in any medium or format, as long as you give appropriate credit to the original author(s) and the source, provide a link to the Creative Commons licence, and indicate if changes were made. The images or other third party material in this article are included in the article's Creative Commons licence, unless indicated otherwise in a credit line to the material. If material is not included in the article's Creative Commons licence and your intended use is not permitted by statutory regulation or exceeds the permitted use, you will need to obtain permission directly from the copyright holder. To view a copy of this licence, visit http://creativecomm ons.org/licenses/by/4.0/.

Funded by SCOAP ${ }^{3}$.

\section{References}

1. D. Glavan, C. Lin, Einstein-Gauss-Bonnet gravity in 4dimensional space-time. Phys. Rev. Lett. 124, 081301 (2020)

2. S. Deser, B. Tekin, Gravitational energy in quadratic curvature gravities. Phys. Rev. Lett. 89, 101101 (2002)

3. S. Deser, B. Tekin, Energy in generic higher curvature gravity theories. Phys. Rev. D 67, 084009 (2003)

4. D. Lovelock, The Einstein tensor and its generalizations. J. Math. Phys. 12, 498-501 (1971)

5. D. Lovelock, The four-dimensionality of space and the Einstein tensor. J. Math. Phys. 13, 874-876 (1972)

6. C. Lanczos, A remarkable property of the Riemann-Christoffel tensor in four dimensions. Ann. Math. 39, 842 (1938)

7. D.G. Boulware, S. Deser, String generated gravity models. Phys. Rev. Lett. 55, 2656 (1985)

8. I. Gullu, T.C. Sisman, B. Tekin, Born-infeld gravity with a unique vacuum and a massless graviton. Phys. Rev. D 92(10), 104014 (2015)

9. R. Bach, Zur Weylschen Relativitätstheorie und der Weylschen Erweiterung des Krümmungstensorbegriffs. Math.Z.9, 110 (1921)

10. J.M.M. Senovilla, Superenergy tensors. Class. Quantum Grav. 17, 2799-2842 (2000)

11. R.B. Mann, S. Ross, The $D \rightarrow 2$ limit of general relativity. Class. Quantum Grav. 10, 1405-1408 (1993)

12. H. Lu, Y. Pang, Horndeski gravity as $D \rightarrow 4$ lmit of Gauss-Bonnet. arXiv:2003.11552 [gr-qc]

13. K. Aoki, M.A. Gorji, S. Mukohyama, A consistent theory of $D \rightarrow$ 4 Einstein-Gauss-Bonnet gravity. arXiv:2005.03859 [gr-qc]

14. D.A. Easson, T. Manton, A. Svesko, $D \rightarrow 4$ Einstein-GaussBonnet gravity and beyond. arXiv:2005.12292 [hep-th] 